\title{
An energy storage device for lifting machinery
}

\section{Wei Han}

School of Mechanical Engineering, North China Electric Power University, Baoding Hebei 071003, China

Keywords: spiral spring; friction clutch; storage box; electromagnetic control; multi-gear unit.

\begin{abstract}
The independent energy storage devices based on spiral spring which has the function of energy storage is used in lift machinery through innovative mechanical design. This device can make maximal use of weight's gravitational potential energy by switching gears to control the amount of energy storage springs. When we lift weights, the stored potential energy will be released in the spindle in order to achieve secondary usage of energy.
\end{abstract}

\section{Development background}

Small light lifting equipment such as electric hoist, when moving cargo, always moves up and down. Upward movement turns electrical energy into potential energy of cargo, while downward movement turns the potential energy into heat energy. The heat energy manly results from the friction between shaftsand wire ropes or chains and gears. Therefore, if the potential energy of cargo can be saved, then the available energy is very significant.

\section{Project signification}

Compared to existing devices, the device proposed in the paper, makes full use of the gravitational potential energy of the goods. According to the difference of weight, it can select the different gears for energy storage which not only ensures the energy efficiency, but also maximize the recovery of gravitational potential energy. The scroll spring can be effectively work in energy conversion, and has good effect on energy saving and emission reduction.

\section{Design scheme}

\subsection{Introduction}

The paper designed a type of auxiliary storage device used for lifting gear. The device is mainly used to control the number of scroll spring. The inner ring of the spindle is fixed on storage box. The scroll spring is driven by power outputting shaft for energy storage. The gear is switched according to cargo weight. When the goods are going down, the motor shaft can drive scroll spring for energy storage. When move upwards, the scroll releases the energy. It is the whole cycle of the device.

\subsection{Product structure and working principle}

Figure 1 shows the principle and instruction of light duty electric hoist hoisting machines. 


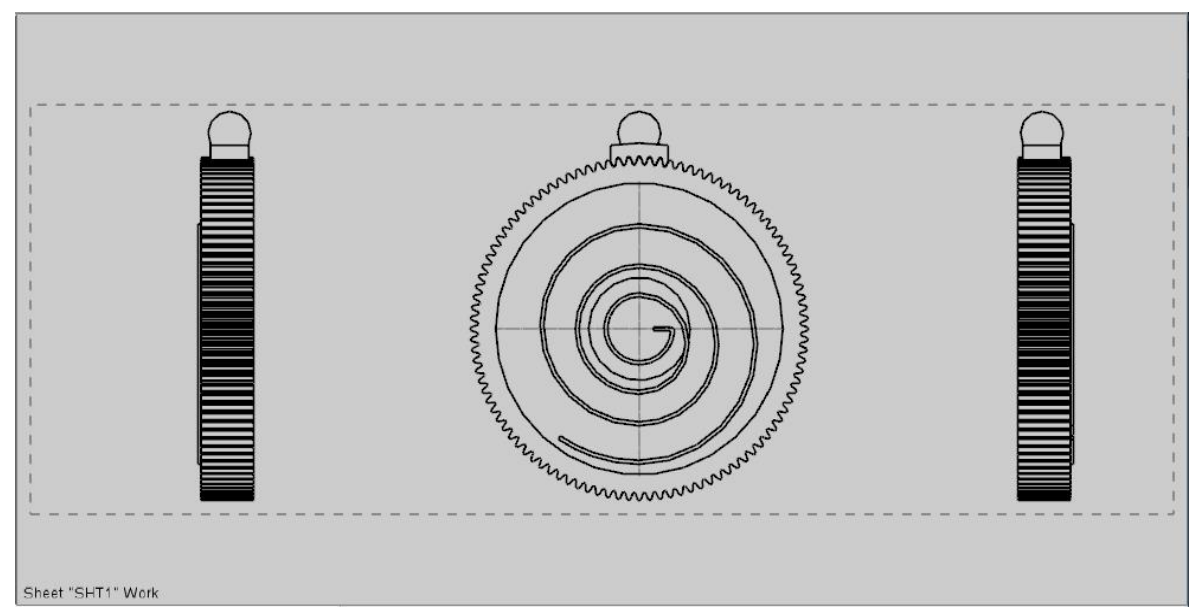

Figure 1 spring box

The device is fixed through a pin.The inner ring of the spindle is fixed on storage box.The outer ring of the spindle is fixed in the spring box.Outside of the spring box, there is gear-like structure. When the switch is on, the gear-like structure engages with the switch and the shaft begin to rotate to save energy.

Through a new type of frictionclutch, the spindle is connected to the power input shaft which is shown in Figure 2. This clutch selects specific friction plate. When scroll spring torsion reaches to appropriate value, the engaged clutch separatesfrom the friction plate and the power is cut from delivering. It can prevent the spring and device from mechanical damage which is caused by spring deformation.

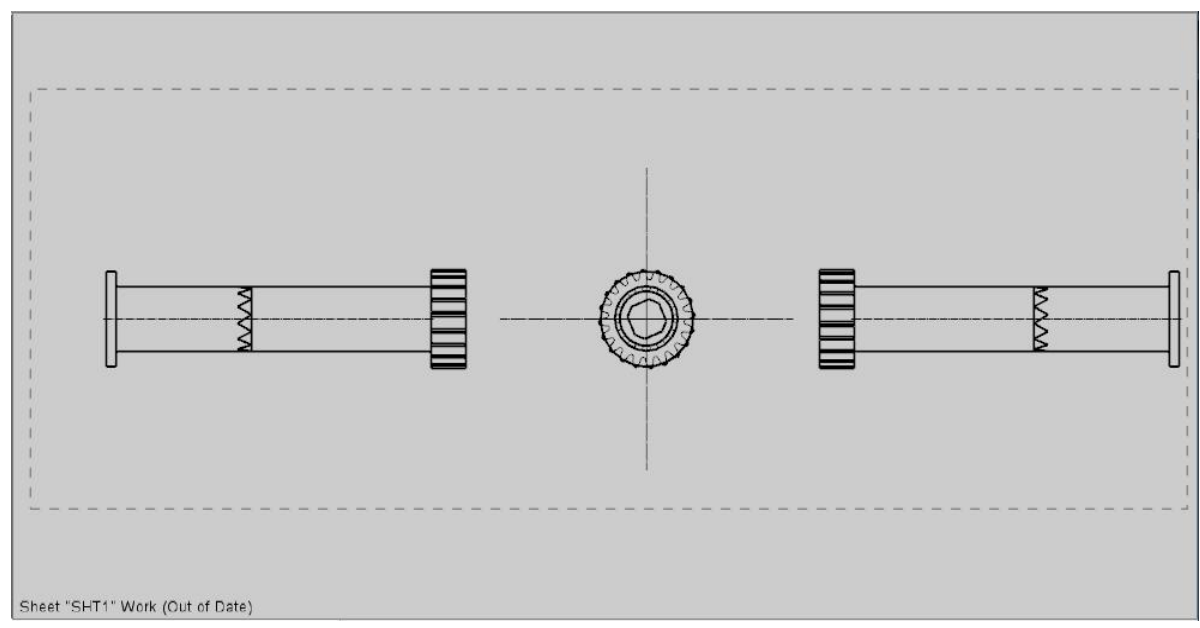

Figure 2 frictionclutch

The design also provides another project based on the number twist of spring for selection. The clutch is designed from grooved pulley. The spindle drives the cylindrical pin and the mouth of the grooved pulley is specified. When the pin swings a certain number of laps, the stop pin of power input shaft goes into the mouth and cut the power off. When we need to access the power again, reverse the power input shaft of same laps and the grooved pulley can be reset. At the same time, the energy stored in a one-way ratchet spring latch is going to prevent the spring from releasing.

When it comes to lifting a heavy object, reset the handle and drive the spindle to move axially. At this time, the clutch is engaged and the power inputs again. The ratchet mentioned above is installed on the spindle between the clutch and spring box. Pawl holdsthe ratchet to prevent the energy-storage spring from releasing on their own. The pawl is controlled by electromagnet which is connected to a switch. When lifting up, the motor start button is pressed and the electromagnet adsorbs the pawl to release the stored energy. When disconnect the motor, the electromagnet is out of power and the pawl falls to carry out prevention work in the next round of the spring release. Under the application of electric hoist, the power source can be gotten from gear box, reel and so on. It is flexible to change the configuration of the device. 


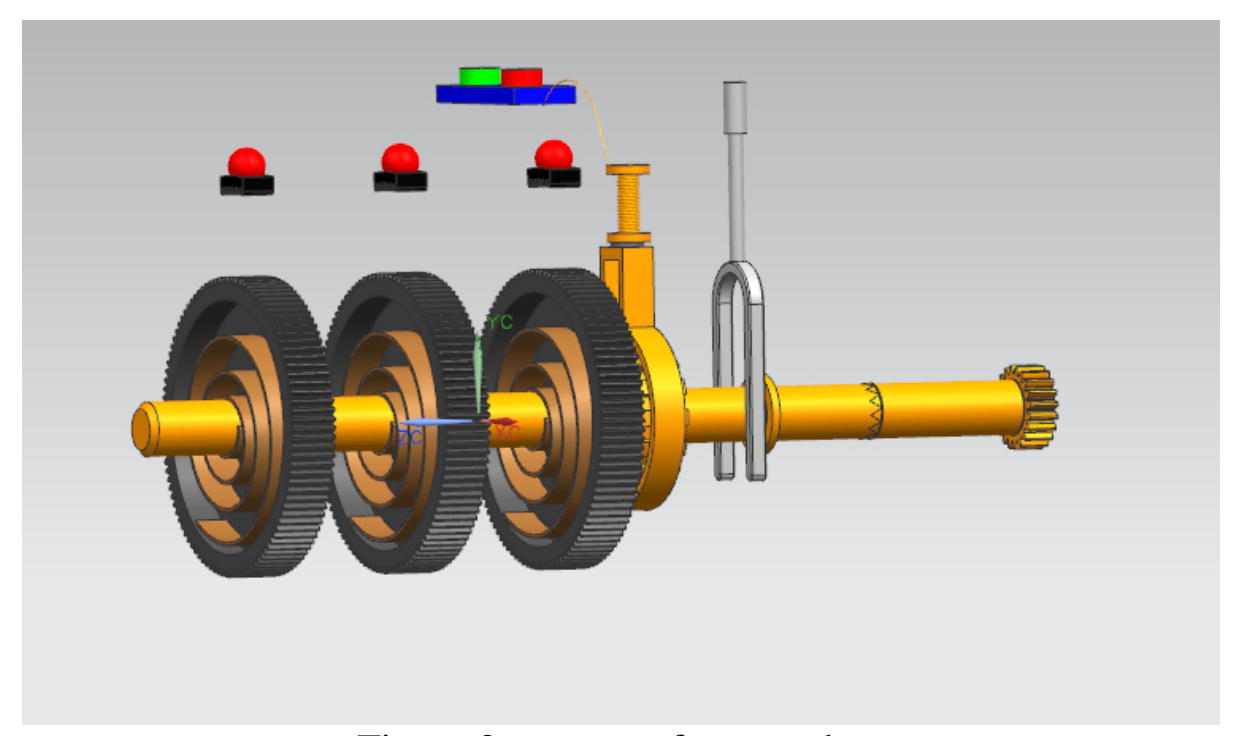

\subsection{Working process}

Figure 3 concept of storage box

When in lifting condition, mounting the weight in electric hoist and the quality can be estimated with different gears. When in descending condition, the spindle begins to rotate and the scroll spring, the spindle is driven to rotate, so does the spring. It changes the gravitational potential energy into a spring elastic potential energy. When the scroll spring rotates to a certain extent, the clutch separates and the energy is saved by the ratchet.

When in lifting condition, mount the weight in electric hoist. The spindle reset and shifting fork pushes the clutch to connect to the power input shaft. When it comes to lifting a heavy object, the power inputs again and the pawl is held up. With the weight rising up, the storage box rotates continuously working as a compensation for the motor.

\section{Innovative analysis}

1. Stall control switch is designed and maximize the energy efficiency of storage

This device uses stall design.According to the difference of weight, it can select the different gears for energy storage. In the traditional structure, the number of scroll springs is invariant and users cannot change it according to the environment. On on hand, few scroll springs are adopted which lead to low energy efficiency. On the other hand, when more scroll springs are involved, it cannot have a good efficiency when the weight is light. The stall design can well solve these kinds of problems. It provides a new way for energy saving.

2. Two clutches are designed which are suitable for different conditions

On project uses a new type of friction clutch. The spindle is connected to the power input shaft. This clutch selects specific friction plate. When scroll spring torsion reaches to appropriate value, the engaged clutch separates from the friction plate and the power is cut from delivering.The design also provides another project based on the number twist of spring for selection. The clutch is designed from grooved pulley which is acentric. When we need to access the power again, reverse the power input shaft of same laps and the grooved pulley can be reset. The two projects have different advantages. The former keeps the continuity while the latter is adjustable. Both of the two projects can keeps high efficiency and protect the device.

3. The design is independent and can be easily installed and removed

As an independent storage device, the scroll spring energy storage devices are more flexible the traditional structure and the maintenance is easy. It greatly improves the reliability and durability. At the same time, it provides the user for expansion. 


\section{Summary}

The device uses independent storage configuration. It can be widely used among different devices. What's more, it is easy to maintain and repair. This unique product design has high efficiency and broad market prospects. It can be considered for mass production. With the rapid development of industry, the device can have a bright future.

\section{References}

[1]. Hengchang Feng. Research on Design and Simulation of Flat Spiral Spring in Energy Storage Equipment [D],North China Electric Power University, China2012. P.6-19.

[2]. Qinfei Huang. The operating device of vortex reed for a braking mechanism[P].China: 101777442, 20091224.

[3]. Guangming Zou, Xiuguan Yang, Chuan Huang et al. Research on the potential energy-driven car with energy stored by vortex coil spring. Mechanical Design. Vol. 29 (2012) No. 4, p. 32-35.

[4]. Wei Duan,Meijiao Liu,Zhangqi Wang et al.A spiral spring energy storage devices for wind turbines[P].China:202579050, 20121205. 